\begin{tabular}{|c|c|c|c|c|c|}
\hline MUNIBE Antropologia-Arkeologia & $n^{\circ} 72$ & $97-110$ & DONOSTIA & 2021 & ISSN 1132-2217 • elSSN 2172-4555 \\
\hline
\end{tabular}

\title{
Secuencias cronológicas del Calcolítico y Edad del Bronce en el interior de la Península lbérica: Análisis de las dataciones de Las Mayores (Numancia de la Sagra, Toledo) en su entorno regional
}

\author{
Chronological sequences of the Copper and Bronze Age in the interior of \\ the Iberian Peninsula: Analysis of the Radiocarbon Dates of Las Mayores \\ (Numancia de la Sagra, Toledo) in their regional surrounding
}

PALABRAS CLAVES: Cuenca del Tajo, campos de hoyos, radiocarbono, análisis bayesiano.

GAKO-HITZAK: Tajo arroa, zulo-zelaia, erradiokarbonoa, analisi bayesiarra.

KEY WORDS: Tagus basin, pits sites, Radiocarbon dates, Bayesian model.

\section{Rosa BARROSO BERMEJO(1), Enrique CERRILLO CUENCA(2), Primitiva BUENO RAMÍREZ(1), Armando GONZÁLEZ MARTíN(3)}

\begin{abstract}
RESUMEN
Analizamos los datos cronológicos del registro funerario del yacimiento de Las Mayores, Toledo, un poblado de estructuras subterráneas que incluyen enterramientos. Las fechas obtenidas en su necrópolis campaniforme certifican la ocupación del enclave durante el III milenio a.C. El resto de los enterramientos se sitúan en el Bronce medio. El modelo bayesiano confirma una interrupción del registro temporal de casi medio siglo, que pone en evidencia las dificultades de interpretar las secuencias de ocupación de estos enclaves considerados como permanentes, con ocupaciones reiteradas o recurrentes en el mismo espacio. Yacimientos equiparables en tipología y decurso cronológico del entorno inmediato no muestran la misma discontinuidad en sus secuencias y sí una cierta contracción en el cambio del III al II milenio a. C. coincidiendo con la emergencia de nuevos poblados lo que sugiere una reorganización poblacional que atenúa la idea de un cambio abrupto en la región.
\end{abstract}

\begin{abstract}
LABURPENA
Toledoko Las Mayoreseko aztarnategiko hilobi-erregistroko datu kronologikoak aztertu ditugu. Aztarnategia ehorzketak biltzen dituzten lurpeko egituren herrixka bat da. Kanpai-forma duen nekropolian egindako azterketen arabera, kokalekua K.a. III. milurtekoan erabili izan zuten. Gainerako ehorzketak Brontze ertainekoak dira. Bayesen ereduak ia mende erdi bateko denbora mailako erregistroaren etena baieztatu du. Horrek agerian uzten du iraunkortzat jotako kokaleku horietako okupazioko sekuentziak interpretatzea zaila dela, espazio berean okupazio errepikakorrak edo behin eta berriz egindakoak gertatu baitziren. Tipologiari eta kronologiari dagokienez berdintsuak diren inguruko aztarnategiek ez dute beren sekuentzietan etenaldi bera erakusten, baina bai nolabaiteko uzkurdura K.a. III. milurtekotik II. milurtekora bitarteko aldaketan. Denbora-tarte hori herrixka berrien beharrarekin bat dator eta horrek iradokitzen du eskualdean aldaketa handia gertatu izanaren ideia arinduko lukeen biztanleriaren berrantolaketa gertatu zela.
\end{abstract}

\begin{abstract}
The main aim of this paper is to analyze the chronological dates of the 3rd and 2nd millennium BC. obtained in the funerary record of Las Mayores, province of Toledo. Simple pits and the absence of complex stratified deposits defined the settlement so that the burials are one of the few primary deposits including in the site. A Bell Beaker necropolis, with several small hypogeum, certifies the occupation of the site during the second half of the 3rd millennium BC. The rest of the identified burials concern to the Middle Bronze and they are scattered throughout all the excavated area. A set of 32 dates from 25 tombs were available and radiocarbon calibration and Bayesian chronological analysis was carried out. The Bayesian approach stress a significant gap of the funerary record between 400-600 years in the period between Copper and Bronze Age. Likewise, the model points out the difficulties of evaluate the sequences of occupation of these sites considered as permanent with repeated occupations over the same location. Although there is a remarkable lack of sites with radiocarbon dates in the middle Tagus basin, at least, three sites close to Las Mayores are similar kind of chronological and cultural context to compare. The summed calibrated radiocarbon date density of them had been compared showing relativity continuity even when in Las Mayores there are not documented burials and new settlements were established in the area. To place Las Mayores in wider regional perspective evidences different population dynamics and suggests to avoid simple interpretations of abrupt abandonment. More dates are necessary in order to provide reliable results in phases of occupation in pits fields.
\end{abstract}

\footnotetext{
(1) Universidad de Alcalá. Facultad de Filosofía y Letras. Área de Prehistoria. C/ Colegios 2, 28801, Alcalá de Henares, Madrid; rosa.barroso@ uah.es; p.bueno@uah.es

(2) Universidad Complutense de Madrid. Facultad de Geografía e Historia. Dpto. de Prehistoria, Historia Antigua y Arqueología. C/ Profesor Aranguren s/n. 28040, Madrid; enriqcer@ucm.es

(3) Universidad Autónoma de Madrid. Facultad de Ciencias. Departamento de Biología. Laboratorio de Poblaciones del Pasado. armando. gonzalez@uam.es
} 


\section{INTRODUCCIÓN}

En el área central de la Península Ibérica los asentamientos característicos durante toda la Prehistoria Reciente, los campos de hoyos, se definen por la agrupación de estructuras negativas excavadas en el subsuelo. Las evidencias arqueológicas de viviendas y fosos (Díaz del Rio, 2003) son reducidas, predominando o apareciendo exclusivamente hoyos que hicieron la función de silos para el almacenaje. Los hoyos se rellenan intencionalmente de diferentes restos secundarios domésticos y en ocasiones enterramientos. Su crecimiento horizontal forma palimpsestos acumulativos (Bayle, 2007) hace años considerados un registro propio de poblaciones no sedentarias y en la actualidad ligados a una movilidad estratégica con ocupaciones recurrentes (Díaz del Rio, 2001; Díaz del Rio y Vicent, 2006). Las fechas son, por tanto, imprescindibles para determinar la continuidad de la ocupación, sin embargo, el área central de la Península Ibérica, especialmente la meseta sur en la que vamos a fijarnos, cuenta con escasos yacimientos prehistóricos del Calcolítico y la Edad del Bronce bien fechados (Balsera y Díaz del Rio, 2014; Balsera et al., 2015a).

En este estudio presentamos la serie de fechas absolutas del Calcolítico y la Edad del Bronce obtenida en los enterramientos del yacimiento de Las Mayores, en el valle del Tajo, con el fin de caracterizar su ocupación. Aunque las fechas son únicamente funerarias no debe invalidarse su aportación, pues es importante señalar que los enterramientos son uno de los pocos depósitos primarios de este tipo de yacimientos. Se intercalan en el espacio doméstico y reaprovechan, como tumba, estructuras de almacenaje previas. Además, uno de los cambios arqueológicos más visibles entre el III y II milenio a.C. es precisamente funerario: la individualidad predominante en los enterramientos de la Edad del Bronce (Balsera et al., 2015a; Valera, 2015), frente a la colectividad anterior.

Para obtener una perspectiva más amplia del registro de Las Mayores, exploramos la cronología de los yacimientos del entorno que cuentan con documentación contrastable, en un tramo del Tajo medio que abarca un área de unos $500 \mathrm{Km}^{2}$ entre Toledo y Madrid. Aquí están los yacimientos con más fechas y más equiparables a Las Mayores en el III y II milenio a.C.

\section{NUEVAS FECHAS PARA EL INTERIOR DE LA PE- NÍNSULA: LAS MAYORES}

El yacimiento de Las Mayores se localiza junto al casco urbano del municipio de Numancia de la Sagra, en la provincia de Toledo (Fig. 1). Ocupa un terreno llano y bien irrigado del valle medio de rio Tajo. En el 2006 se excavaron 2,46 H que confirman su adscripción como poblado abierto formado exclusivamente por estructuras negativas. No conocemos su extensión completa, pero hasta el momento el empleo de técnicas de teledetección no ha mostrado la existencia de fosos o cualquier otra delimitación estructural del enclave. Sus
605 estructuras, de las que se excavó el 76\% (Perea et al. 2010), corresponden exclusivamente a hoyos, sin localizarse vivienda alguna. Estaban colmatadas con restos secundarios de fauna, cerámica y, en ocasiones, enterramientos. 27 estructuras, hoyos y pequeños hipogeos de doble cámara, fueron utilizados como tumbas. Una tumba más, identificada en los trabajos de campo con un fragmento de hueso humano, no ha podido ser confirmada finalmente.

Los análisis antropológicos determinan el enterramiento de 52 individuos. Prácticas funerarias, ajuares y restos materiales muestran dos tipos distintos de enterramientos. Al III milenio cal BC. y ámbito campaniforme, hay que asociar 7 tumbas reunidas en un pequeño espacio central de $150 \mathrm{~m}^{2}$ : Se trata de una fosa y seis pequeños hipogeos con piedras de cierre que acogen enterramientos individuales y colectivos de hasta 6 individuos. Mayoritariamente tienen ajuares destacados con cerámica, lisa o decorada, punzones de cobre, brazales de arquero o botones, y los cuerpos fueron cubiertos con cinabrio (Barroso et al., 2018a). Este mineral, localizado a unos $150 \mathrm{~km}$ del yacimiento, o el marfil, sugieren comunidades implicadas en largas redes de intercambio. Una única tumba en hoyo simple carece de cinabrio y elementos de ajuar, aunque es contemporánea y contigua al resto (Fig. 2).

Los enterramientos de la Edad del Bronce (20 tumbas con un total de 31 individuos) se distribuyen por toda el área excavada en hoyos simples, tres de ellos además en vasijas (Barroso et al., 2018b). Son mayoritariamente individuales y excepcionalmente cuentan con algún ajuar u ofrenda animal. La cerámica Cogotas I, característica del interior peninsular entre el 1800-1150 cal BC (Barroso et al., 2014; Esparza et al., 2012) aparece usualmente entre los restos secundarios del relleno de las tumbas. El cinabrio no está presente en ninguno de los enterramientos y no hay evidencias de intercambios de larga distancia. Todo sugiere que la riqueza de su comunidad reside en bienes primarios, en pocas ocasiones utilizados en las tumbas para distinguir unos de otros.

El análisis osteológico permitió determinar el NMI de cada tumba y la muestra de hueso seleccionada para fechar. La selección de muestras se centró en fechar el mayor número de tumbas. En las tumbas colectivas, con varios eventos funerarios, fechamos el último enterramiento, previo al cierre de la estructura, y los individuos con suficiente hueso preservado. Preferentemente se seleccionaron fragmentos de cráneo. En total se muestrearon 25 tumbas obteniéndose dataciones radiométricas para 32 individuos. Las dos tumbas restantes, por sus cerámicas decoradas, pueden adscribirse claramente a la Edad del Bronce.

Todas las fechas han sido obtenidas por AMS en el laboratorio de Beta Analytic, Miami. Su identificación consta del código que proporciona el laboratorio, junto al número de tumba y entre paréntesis el individuo, en el caso de haber más de uno. Las fechas han sido calibradas mediante el paquete estadístico Bchron (Haslett 

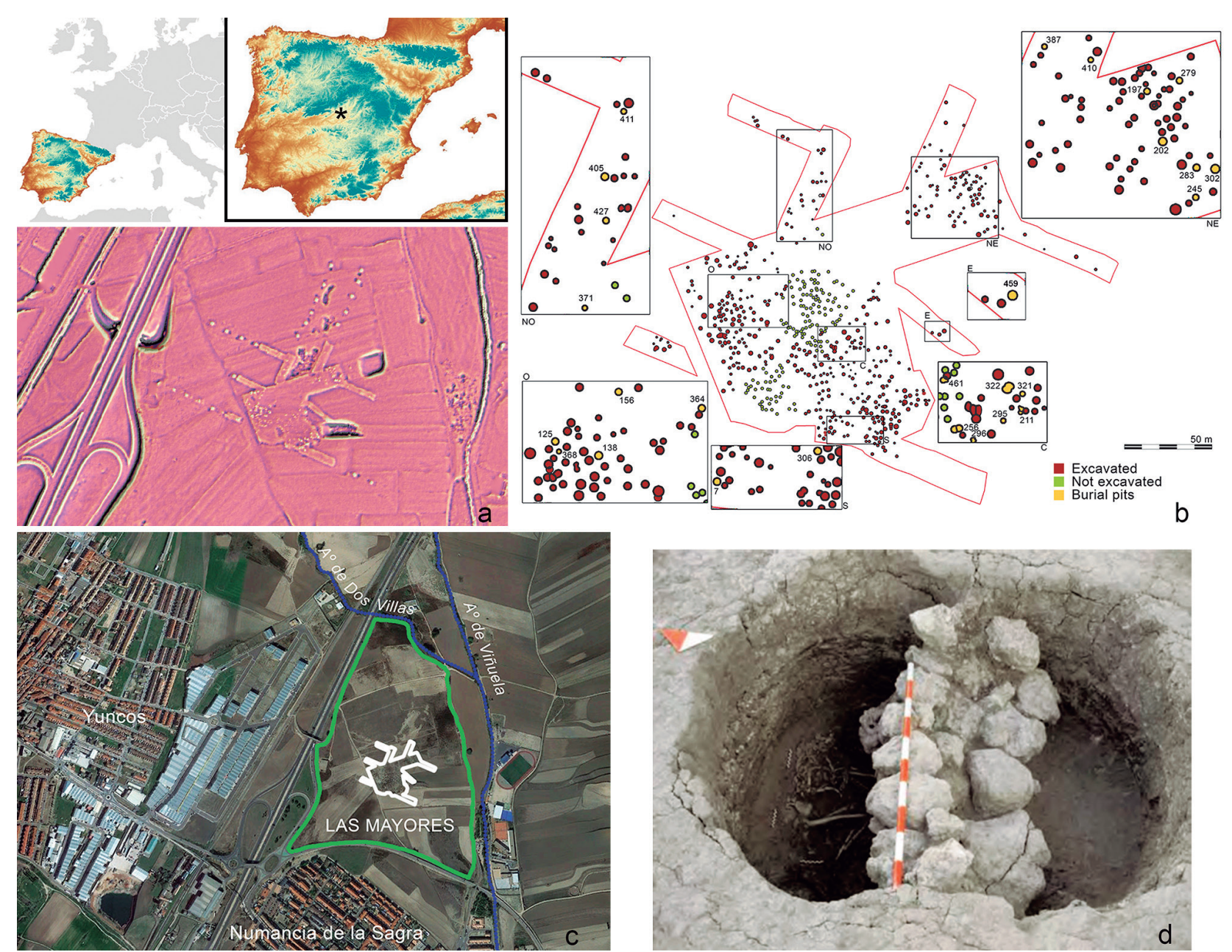

Fig.1. Las Mayores, Numancia de la Sagra (Toledo): a) Imagen derivada de datos LIDAR del área del yacimiento; b) Plano del área excavada con la situación de los enterramientos; c) Foto área con la situación del yacimiento al norte del término de Numancia de la Sagra (fuente SIGPAC) Ministerio de Agricultura, pesca y alimentación; d) Foto de una tumba campaniforme del yacimiento. / Las Mayores, Numancia de la Sagra (Toledo): a) LIDAR-derived image of the site; b) Plan of excavated area with the location of the burials; c) Location of de prehistoric site from Agricultural Plots Geographic Information System (SIGPAC) of Ministry of Agriculture, Fisheries and Food; d) Photo of a Bell Beaker tomb of the site.

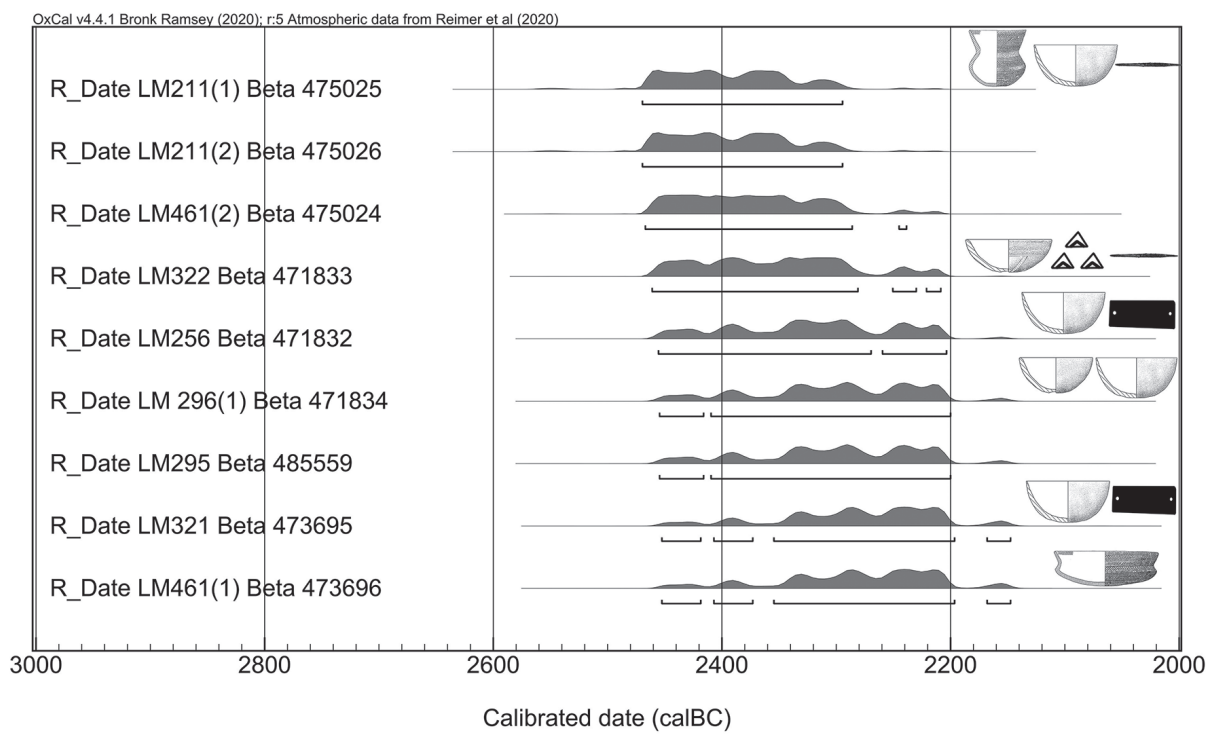

Fig.2. Fechas calibradas de las tumbas de la necrópolis campaniforme de Las Mayores, Numancia de la Sagra (Toledo) con esquema de su ajuar. / Calibrated radiocarbon dates of Bell Beaker tombs from Las Mayores, Numancia de la Sagra (Toledo) with the grave goods. 


\begin{tabular}{|c|c|c|c|c|}
\hline Tumba & Código LAB. & C:N & $\begin{array}{l}\text { Determinación radiocarbónica } \\
\text { BP y desviación estándar }\end{array}$ & Intervalos calibrados cal BC con $2 \sigma$ de probabilidad \\
\hline LM.211 (1) & Beta-475025 & 3.3 & $3910 \pm 30$ & $2467-2296(94.3 \%)$ \\
\hline LM.211 (2) & Beta-475026 & 3.3 & $3910 \pm 30$ & 2467-2296 (94.3\%) \\
\hline LM.461(2) & Beta-475024 & 3.3 & $3890 \pm 30$ & 2464-2287- (93.6\%), 2243-2238 (0.8\%), \\
\hline LM.322 & Beta-471833 & 3.3 & $3870 \pm 30$ & $2459-2280$ (87\%), 2250-2229 (5.1\%), 2220-2208 (2.3\%) \\
\hline LM.256 & Beta-471832 & 3.2 & $3850 \pm 30$ & 2454-2414 (24.8\%), 2409-2269 (57.3\%), 2258-2204 (12.4\%) \\
\hline LM.296 (1) & Beta-471834 & 3.3 & $3840 \pm 30$ & $2453-2417$ (23.2\%), 2407-2369 (21.6\%), 2364-2200 (49.5\%) \\
\hline LM.295 & Beta-485559 & 3.3 & $3840 \pm 30$ & $2453-2417$ (23.2\%), 2407-2369 (21.6\%), 2364-2200 (49.5\%) \\
\hline LM. 321 & Beta-473695 & 3.3 & $3830 \pm 30$ & $2451-2419(23.1 \%), 2406-2374(20.8 \%), 2352-2197(48 \%), 2168-2148(2.4 \%)$ \\
\hline LM: 461(1) & Beta-473696 & 3.3 & $3830 \pm 30$ & $2451-2419(23.1 \%), 2406-2374$ (20.8\%), 2352-2197 (48\%), 2168-2148 (2.4\%) \\
\hline LM.459(4) & Beta-419745 & * & $3370 \pm 30$ & $1740-1707$ (29.8\%), 1699-1599 (56.3\%), 1589-1588 (0.5\%), 1585-1543 (7.6\%) \\
\hline LM.138 & Beta-503393 & 3.3 & $3350 \pm 30$ & $1734-1716(15.7 \%), 1690-1535(78.4 \%)$ \\
\hline LM.459(1) & Beta-419744 & * & $3340 \pm 30$ & $1732-1717(14.1 \%), 1688-1532(80 \%)$ \\
\hline LM.411 & Beta-500177 & 3.3 & $3340 \pm 30$ & $1732-1717(14.1 \%), 1688-1532(80 \%)$ \\
\hline LM.283(1) & Beta-413610 & * & $3340 \pm 30$ & $1732-1717(14.1 \%), 1688-1532(80 \%)$ \\
\hline LM.283(2) & Beta-419743 & * & $3310 \pm 30$ & $1666-1655(11.4 \%), 1633-1504$ (82.6\%) \\
\hline LM.410 & Beta-497404 & 3.3 & $3310 \pm 30$ & $1666-1655(11.4 \%), 1633-1504$ (82.6\%) \\
\hline LM.387 (1) & Beta-503392 & 3.3 & $3310 \pm 30$ & $1666-1655(11.4 \%), 1633-1504$ (82.6\%) \\
\hline LM283(4) & Beta-410104 & 3.2 & $3300 \pm 30$ & $1624-1501(94 \%)$ \\
\hline LM.302 & Beta-420953 & * & $3260 \pm 30$ & $1610-1571$ (48.9\%), 1567-1491 (36.4\%), 1481-1449 (8.3\%) \\
\hline LM-405 & Beta-413611 & * & $3260 \pm 30$ & $1610-1571(48.9 \%), 1567-1491$ (36.4\%), 1481-1449 (8.3\%) \\
\hline LM-245 & Beta- 418600 & * & $3250 \pm 30$ & $1608-1574$ (44.4\%), 1560-1553 (7.3\%), 1545-1440 (41.8\%) \\
\hline LM.202 & Beta-419742 & * & $3250 \pm 30$ & $1608-1574$ (44.4\%), 1560-1553 (7.3\%), 1545-1440 (41.8\%) \\
\hline LM.197 & Beta-503390 & 3.3 & $3240 \pm 30$ & $1606-1604$ (4.7\%), 1600-1583 (23\%), 1558-1555 (4.8\%), 1543-1431 (61\%) \\
\hline LM.125 & Beta-420955 & * & $3240 \pm 30$ & $1606-1604$ (4.7\%), 1600-1583 (23\%), 1558-1555 (4.8\%), 1543-1431 (61\%) \\
\hline LM.368 & Beta-497405 & 3.3 & $3240 \pm 30$ & $1606-1604$ (4.7\%), 1600-1583 (23\%), 1558-1555 (4.8\%), 1543-1431 (61\%) \\
\hline LM.279 & Beta-500179 & 3.2 & $3230 \pm 30$ & $1594-1594(1.5 \%), 1537-1424(91.9 \%)$ \\
\hline LM. 371(2) & Beta-492811 & 3.3 & $3200 \pm 30$ & $1507-1416(93.7 \%)$ \\
\hline LM.427 & Beta-420954 & * & $3200 \pm 30$ & $1507-1416(93.7 \%)$ \\
\hline LM.156 & Beta-500178 & 3.2 & $3180 \pm 30$ & $1502-1407(93.8 \%)$ \\
\hline LM. 371(1) & Beta-536648 & 3.3 & $3160 \pm 30$ & $1498-1389(90.6 \%), 1334-1322(3 \%)$ \\
\hline LM.387 (2) & Beta-505894 & 3.3 & $3160 \pm 30$ & $1498-1389(90.6 \%), 1334-1322(3 \%)$ \\
\hline LM.364 (1) & Beta-492810 & 3.3 & $3140 \pm 30$ & $1494-1469(34.8 \%), 1464-1377(50.7 \%), 1343-1305(8 \%)$ \\
\hline
\end{tabular}

Tabla 1: Dataciones absolutas del sitio de Las Mayores. Todas las dataciones se han realizado a partir de colágeno. La calibración se ha realizado con el software Bchron y la curva atmosférica IntCal20. / Absolute dates on bone collagen from Las Mayores site. All the dates have been calibrated with Bchron software and the atmospheric curve IntCal20.

* No proporcionado por el laboratorio.

y Parnell, 2008) para el lenguaje de programación R y la curva IntCal20 (Reimer et al., 2020).

\section{EL CONTEXTO DE COMPARACIÓN}

Sólo 5 yacimientos distribuidos a lo largo de la cuenca media del Tajo y sus afluentes, incluido Las Mayores, tienen más de 10 fechas para el III y II milenio cal BC. Dejando la cueva funeraria del Rebollosillo (Diaz del Rio et al., 2017a) en la sierra madrileña, otros tres yacimientos madrileños, Camino de las Yeseras, Humanejos y Los Berrocales, son comparables con Las Mayores. Coinciden con él en ser poblados de la misma tipología, palimpsestos de estructuras, en ubicarse en la misma unidad geográfica y en tener registro funerario datado superpuesto al área doméstica. Además, las fechas son, en su mayoría, de muestras de vida corta y en dos de ellos proceden del ámbito doméstico y funerario. (Fig. 3)

Camino de las Yeseras (Madrid) es un poblado de fosos de 80 ha (Balsera et al., 2015a: 151) en la confluencia de los ríos Henares y Jarama, cerca de la ciudad de Madrid. Está formado por cinco fosos concéntricos, una zanja excéntrica y más de 8500 estructuras de las que se han excavado un 16\% (Arteaga et al., 2017). Los enterramientos asociados al poblado comprenden 
inhumaciones colectivas y tumbas campaniformes más complejas que abarcan toda la secuencia. El yacimiento se ocupa intensamente durante el III milenio cal BC, se abandona a comienzos del II cal BC y recibe de nuevo en el Bronce medio una pequeña ocupación (Blasco et al., 2007). Sus fosos se amortizan no mucho más allá de mediados del III milenio cal BC a la vez que se intensifica la actividad funeraria (Balsera et al., 2015a: 153).

Es el yacimiento mejor fechado de la Comunidad de Madrid con 55 fechas, 33 (5 de ellas sobre carbón) proceden de contextos domésticos, recintos, viviendas o depósitos con restos animales y 22 (todas ellas en hueso) de contextos funerarios (Blasco et al., 2016; Blasco y Liesau, 2019; Olalde et al., 2018 y 2019; Ríos, 2011). De ellas, 8 proceden de enterramientos asociados a campaniforme cuya cronología alcanza en el yacimiento las fechas más recientes de la secuencia regional en el 1922-1560 cal BC, un intervalo de probabilidad estimado al 68\% por un modelo bayesiano (Blasco et al., 2019: 250). El resto son enterramientos colectivos e individuales fuera de esta asociación. En total, se trata de una veintena de individuos frente al medio centenar no asociado a campaniforme (Liesau et al., 2018: 143). Un enterramiento más corresponde a la etapa Protocogotas (Blasco et al., 2007).

Humanejos es un poblado madrileño localizado en una loma del término de Parla, junto al arroyo de Huma- nejos. Tiene de más de 20 ha. de extensión sin que haya llegado a delimitarse por completo. Se localizaron 2405 estructuras de las que se excavan 2391, incluyendo un pequeño foso de $0,12 \mathrm{H}$. Su cronología prehistórica se extiende desde finales del IV milenio cal BC, hasta la Edad del Hierro siendo la ocupación calcolítica la mejor representada. Al registro doméstico se superpone un notable conjunto funerario con 106 tumbas y 168 individuos enterrados durante toda la secuencia. Las fechas plantean la ocupación prácticamente continua del enclave durante 2000 años (Garrido-Pena et al., 2019).

Del yacimiento, conocemos hasta el momento $24 \mathrm{fe}$ chas de distintos laboratorios, todas ellas en hueso. Proceden de 18 tumbas, 9 de ellas con Campaniforme de una quincena, como mínimo, de individuos (Garrido-Pena et al., 2019). La modelación bayesiana de las fechas de estos últimos enterramientos permite fijar su comienzo y final entre el 2512-2447 y el 2194-2077 (con un 68\% de probabilidad) (García y Tejedor, 2019). No se han incluido las fechas duplicadas en carbón y hueso de la misma estructura (Ríos, 2011: 83) y de las realizadas sobre el mismo individuo tomamos una combinación de sus distribuciones de probabilidad antes de calibrarlas.

Los Berrocales es un yacimiento madrileño localizado cerca del interfluvio de los ríos Jarama y Henares, sobre el pequeño cerro del Alto de las Peñuelas, a unos $5 \mathrm{Km}$ de Camino de las Yeseras. Tiene cerca de 20

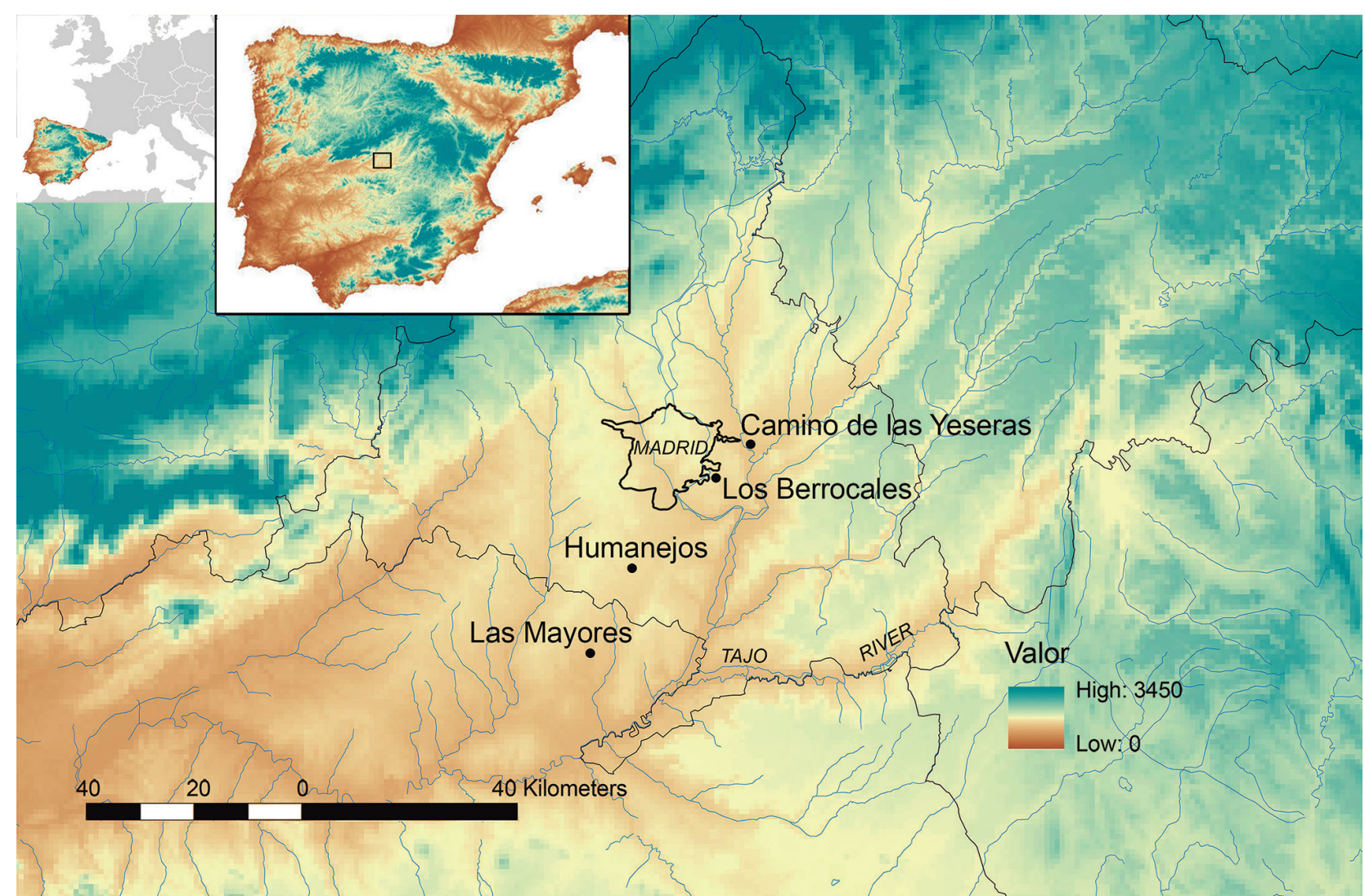

Fig.3. Mapa con la situación del área con los yacimientos analizados en el texto./ Map of the area and the sites analysed in the text. 
ha. de extensión y 2000 estructuras que forman cuatro agrupaciones diferenciables separadas por algo más de 100 m. y sin ninguna delimitación. En ellas se encontró una única vivienda y restos de 52 individuos en 40 tumbas en forma de hoyos excavados en el suelo. Del yacimiento se han obtenido 20 fechas, 13 de ellas de enterramientos y el resto, de estructuras domésticas. Ha sido adscrito a una ocupación de nueva planta que se extiende desde el Bronce antiguo hasta el final del Bronce pleno, con una única fecha del Bronce final (Aliaga y Mejías, 2011; Pérez Villa, 2015).

Los cuatro yacimientos reúnen 131 dataciones de un total de 264 para la Comunidad de Madrid, la provincia de Toledo y Guadalajara'. A las 152 recogidas recientemente para III y II milenio cal BC en la zona (Balsera et al., 2015a) hay que sumar las obtenidas posteriormente (Barroso et al., 2014; Blasco et al., 2019; Díaz del Rio et al., 2017a; Galindo et al., 2018; Pérez Villa, 2015: 263). El material suplementario se puede consultar en el siguiente link.

\section{MÉTODOS}

Uno de los problemas en el desarrollo de las interpretaciones de los sitios es la detección de fases. La aproximación cuantitativa más habitual en los últimos años ha consistido en el diseño de modelos bayesianos (Buck y Meson, 2015), que tratan de contrastar estadísticamente asunciones previas sobre el conjunto de dataciones. En este trabajo hemos tratado de ofrecer una doble alternativa mediante la creación de modelos cronológicos basados en la suma de la densidad de las dataciones. En primer lugar, hemos realizado un modelo bayesiano para las cronologías de la necrópolis de Las Mayores con el objetivo de dilucidar las etapas de uso de las sepulturas distribuidas en el yacimiento. En segundo lugar, hemos realizado una comparación de las densidades de probabilidad de las dataciones de los 4 yacimientos estudiados, para tratar de observar tendencias en sus fases de ocupación. Este último análisis tiene por tanto una finalidad exploratoria.

\subsection{El modelo bayesiano para el sitio de Las Mayores}

El modelo se realizó empleando el software BCal (Buck et al., 1999). Cada individuo fue considerado un evento concreto $(\mathrm{x})$ al que se asignaron las distribuciones de probabilidad de las dataciones $(\theta)$. El modelo bayesiano se construyó teniendo en cuenta tanto las dataciones como los ajuares asociados a los individuos. Siguiendo las recomendaciones del software, todos los resultados a posteriori se ofrecen redondeados a la fecha más próxima de su intervalo a 10 años.

La información a priori está constituida por el conjunto de eventos organizados en dos fases sin continuidad entre ellas, según lo observado en el registro. Dado que las fases no son contiguas se establecieron límites (boundaries en la bibliografía inglesa para cada una de las dos fases). La primera fase aglutinaba a las tumbas campaniformes y la segunda a las identificadas como pertenecientes a la Edad del Bronce (Tabla 2).

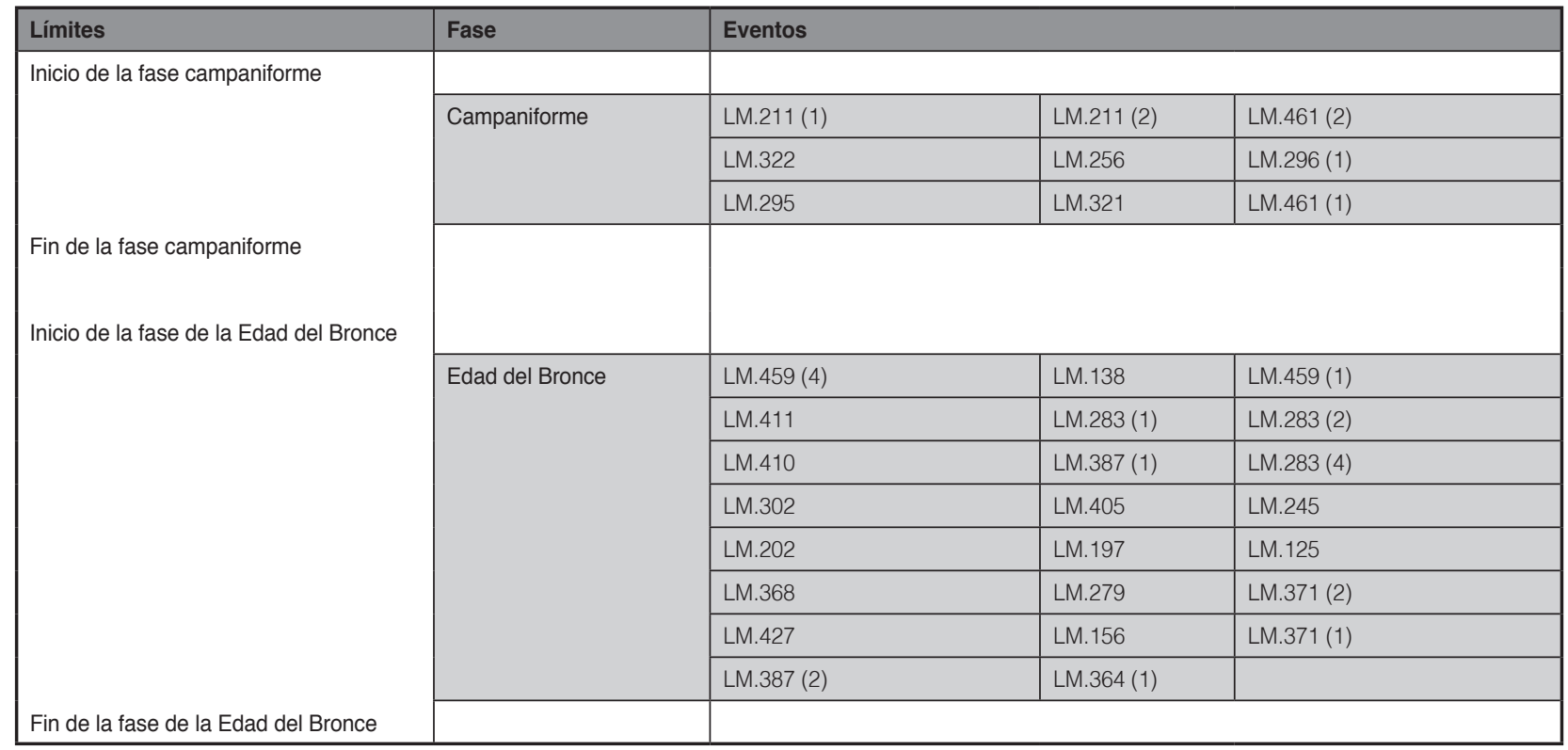

Tabla 2: Elementos que conforman la información a priori introducida en el modelo bayesiano. / Information a priori introduced in the Bayesian model.

\footnotetext{
1 Todas las fechas se incluyen como material suplementario. Se ha optado por la circunscripción administrativa para conseguir una delimitación precisa. Las fechas de Guadalajara proceden de su ámbito occidental, dentro del cauce medio del Tajo.
} 
Dada la nitidez con la que se reconocieron las fechas no se consideró necesario el diseño de un modelo alternativo.

Adicionalmente se analizaron los resultados de las densidades de probabilidad a posteriori para aquellas sepulturas con más de un evento (individuo datado) con el objetivo de tratar de reconocer reutilizaciones o reaperturas de estos contextos para inhumar a nuevos individuos.

\subsection{Modelos de densidad de la probabilidad para el conjunto de sitios}

Los modelos de densidad basados en suma de probabilidades (SPD) han tenido relevancia en el análisis de las fluctuaciones demográficas en la Prehistoria peninsular (Balsera et al., 2015b; Bernabeu et al., 2016,) pero en este caso se presenta una solución similar adaptada a la problemática de varios sitios para evaluar las secuencias cronológicas a una escala espacial más reducida. Hemos aplicado una metodología basada en la mezcla gaussiana de las estimaciones de la densidad de probabilidad, que ya se ha empleado con éxito en otros ámbitos (d'Alpoim Guedes et al., 2016; Orton et al., 2016). Se ha utilizado esta herramienta con un fin exploratorio de las secuencias cronológicas que puedan existir en una escala regional. Hemos implementado este modelo mediante la biblioteca Bchron (Haslett y Parnell, 2008) para el lenguaje de programación $\mathrm{R}$, y en concreto la función BchronDensity. Esta función ajusta un modelo de fases no paramétrico, empleando la estimación de la densidad de probabilidad a partir de la mezcla de distribuciones gaussianas (gaussian mixture density estimation) (McLachlan y Peel, 2000) de cada yacimiento.

Para ello se parte de n distribuciones (dataciones) y la siguiente fórmula:

$$
p(x)=\sum_{s=1}^{n} \pi_{s} p\left(x ; \theta_{s}\right)
$$

Donde $p(x)$ es una función que expresa la probabilidad de que un evento $x$ ocurra en un determinado punto de la secuencia. Con esta técnica de reducción de la dimensionalidad se obtiene una distribución de probabilidad para el conjunto de los $n$ eventos observados a lo largo de la secuencia. Como en cualquier interpretación de la densidad de la probabilidad, se debe tener en cuenta que el área total es siempre 1 y que deben establecerse límites significativos para la interpretación.

El ajuste de estos límites permite interpretar fases dentro de la secuencia general. Este es el objetivo de la metodología que presentamos, el detectar hiatus cuyas densidades en puntos concretos de la curva (cronología), desciendan de un nivel de probabilidad dado.
Finalmente, debemos indicar que en el conjunto de datos contamos con 4 individuos del sitio de Humanejos con más de una datación (Blasco et al., 2016; Garrido-Pena et al., 2019). Con el objetivo de no introducir redundancias hemos optado por combinar las distribuciones de probabilidad de las dataciones antes de realizar la calibración. El cálculo de $\chi^{2}$ revela, en los 4 casos, un estadístico inferior a 3,8, por lo que puede considerarse que las medias proceden de distribuciones similares con un margen de error inferior al $5 \%{ }^{2}$. El resultado de la combinación se ha calibrado, finalmente, como si se tratara de una única datación. Esta técnica, sugerida por Shennan (1988: 65), permite obtener una única distribución del elemento datado con una menor varianza.

\section{RESULTADOS}

\subsection{Un modelo cronológico bayesiano para Las Mayores}

Según las recomendaciones de uso de BCal, se comprobó la convergencia del modelo ejecutándolo en tres ocasiones empleando una semilla aleatoria. La diferencia máxima obtenida entre los tres modelos fue de 6 años, dato que prueba un óptimo diseño. Con el mismo propósito y de forma alternativa se empleó la biblioteca ArchaeoPhases (Philippe y Vibet, 2020). Se comprobó que las cadenas de Markov alcanzaron equilibrio mediante distintas pruebas estadísticas.

Las 32 fechas radiocarbónicas de Las Mayores cubren el 92,5\% de las tumbas localizadas. La desviación estándar de las dataciones es de 30 años, siendo constante a lo largo de toda la serie. Aunque en realidad el laboratorio ajusta a esa cantidad cualquier desviación inferior por cuestiones técnicas, la escasa magnitud de este valor permite mejorar la resolución final del modelo reduciendo la incertidumbre. Según la información a posteriori que devuelve el modelo realizado, el enclave ha sido utilizado como lugar de enterramiento entre el lapso definido entre 2490-1400 cal BC (95\%), es decir durante un periodo amplio que abarca Calcolítico y Edad del Bronce. Sin embargo, las fechas no delimitan una serie continua sino intervalos temporales cuyo solape necesita una lectura de detalle.

Teniendo en cuenta los resultados del modelo bayesiano, los valores que definen los límites de las fases pueden estimarse con un umbral que represente un porcentaje significativo del área de la distribución de densidad a posteriori que devuelve el modelo. Ofrecemos en la tabla 3 los resultados del modelo con un 95\% de probabilidad, estimada a partir del área de mayor densidad de la probabilidad y adicionalmente con un $80 \%$, como ha sido empleado en otros trabajos (Jover Mestre et al., 2014). De forma errónea se toma como

\footnotetext{
${ }^{2}$ Los resultados se incluyen como material suplementario.
} 


\begin{tabular}{|l|l|l|}
\hline Intervalo & Límite del MDP (95\%) & Límite del MDP (80\%) \\
\hline Inicio de la fase campaniforme & $2490-2300 \mathrm{cal} \mathrm{BC}$ & $\begin{array}{l}2440-2420 \mathrm{cal} \mathrm{BC} \\
2420-2380 \mathrm{cal} \text { BC } \\
2380-2320 \mathrm{cal} \mathrm{BC}\end{array}$ \\
\hline Fin de la fase campaniforme & $\begin{array}{l}2400-2360 \mathrm{cal} \mathrm{BC} \\
2340-2170 \mathrm{cal} \mathrm{BC}\end{array}$ & $2340-2230 \mathrm{cal}$ BC \\
\hline Inicio de la fase de la Edad del Bronce & $1650-1550 \mathrm{cal} \mathrm{BC}$ & $1630-1570 \mathrm{cal} \mathrm{BC}$ \\
\hline Fin de la fase de la Edad del Bronce & $1480-1400 \mathrm{cal} \mathrm{BC}$ & $1470-1410 \mathrm{cal}$ BC \\
\hline
\end{tabular}

Tabla 3: Límites del área de Mayor Densidad de la Probabilidad (MDP) a posteriori para las fases analizadas en Las Mayores. / A posteriori Higtest Density Probability Area for the phases analyzed at Las Mayores. límites para estos umbrales el porcentaje de la desviación estándar en una distribución normal o dos veces su valor. Esta elección, propia de la estadística inferencial, no resulta posible en los modelos bayesianos ( $\mathrm{Ha}-$ milton y Krus, 2018: 195-196; Ramsey, 2009: 353-354). En este trabajo nos decantamos por este último umbral para caracterizar los intervalos cronológicos por mantener un nivel razonable entre precisión y probabilidad.

El modelo estima un inicio de la fase campaniforme en el intervalo 2440-2320 cal BC y un fin en el intervalo 2340-2230 cal BC (Fig. 4). Este solapamiento se debe a un uso funerario del emplazamiento relativamente rápido, y en el que, además, deben tener influencia las mesetas características de la curva de calibración, como ya se ha advertido para otros yacimientos de la región (García y Tejedor 2019: 323). La duración estimada a posteriori del intervalo entre la fase de inicio y la final muestra, por tanto, el mismo grado de imprecisión: de 200 a 460 años. El límite inferior (200 años) confirmaría la recurrencia del lugar de enterramiento de una forma episódica, si bien hay que advertir que esta interpretación está limitada por lo conocido en la superficie excavada del sitio. (Tabla 4. Fig. 5)
En la fase campaniforme las dataciones radiocarbónicas sitúan el último enterramiento, pero el escaso número de fechas obtenidas dificulta establecer el periodo de uso de la construcción en aquellas que son colectivas. El análisis antropológico de la tumba más numerosa, LM.296, determina varios individuos únicamente por piezas dentales incompatibles con el resto de los esqueletos. Su preservación las convierte en la única evidencia de las diferentes remociones realizadas en la tumba. No descartamos que igual que en otros contextos colectivos (Aranda et al., 2020) sean pruebas de un mayor periodo de uso de los hipogeos y quizás pudieran remontar las fechas de la construcción y del yacimiento.

Para analizar esta cuestión, las tumbas con más de un individuo datado han sido analizadas a posteriori empleando las capacidades del software BCal, que permite estimar en términos de probabilidad la relación cronológica entre las dataciones que representan a dos eventos. La probabilidad más baja es la de la sepultura LM.211, con un 50\% de probabilidad de que los individuos se hubieran depositado en momentos distintos. En el contexto de LM.461 la probabilidad es más ele-

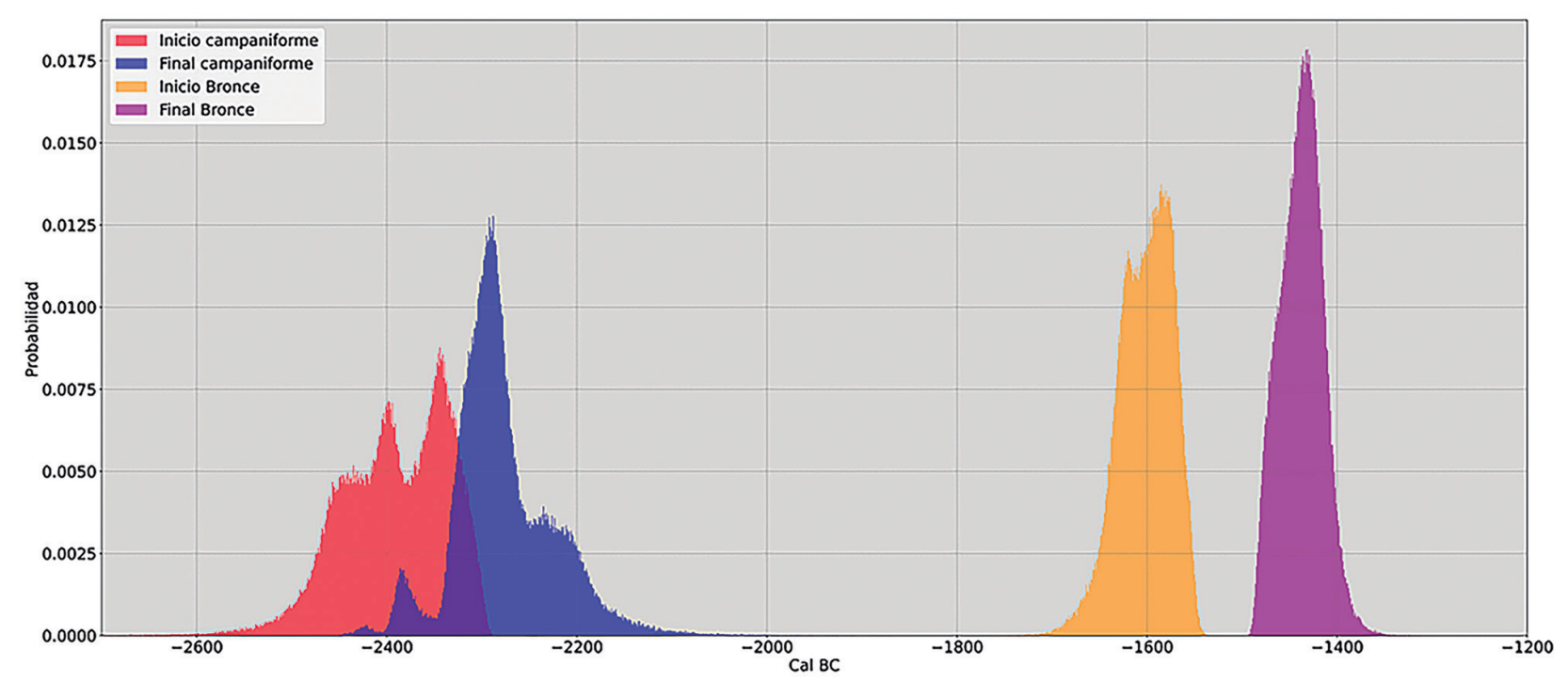

Fig.4. Gráfico de densidad de la probabilidad a posteriori para cada uno de los límites cronológicos. / Posterior probability density plot for the modelled limits of each phase. 


\begin{tabular}{|l|l|l|}
\hline Tiempo transcurrido entre límites & Límite del MDP (95\%) & Límite del MDP (80\%) \\
\hline $\begin{array}{l}\text { Desde el inicio de la fase campaniforme } \\
\text { al final de la fase campaniforme }\end{array}$ & 140 - 590 años & $200-460$ años \\
\hline $\begin{array}{l}\text { Desde el fin de la fase campaniforme al } \\
\text { inicio de la fase de la Edad del Bronce }\end{array}$ & $\begin{array}{l}310-320 \text { años } \\
330-680 \text { años }\end{array}$ & $410-640$ años \\
\hline $\begin{array}{l}\text { Desde el fin de la fase campaniforme al } \\
\text { inicio de la fase de la Edad del Bronce }\end{array}$ & $80-240$ años & $110-220$ años \\
\hline
\end{tabular}

Tabla 4: Duración de los intervalos a posteriori para la secuencia de enterramientos de Las Mayores. / A posteriori intervals for the Las Mayores burials sequence.
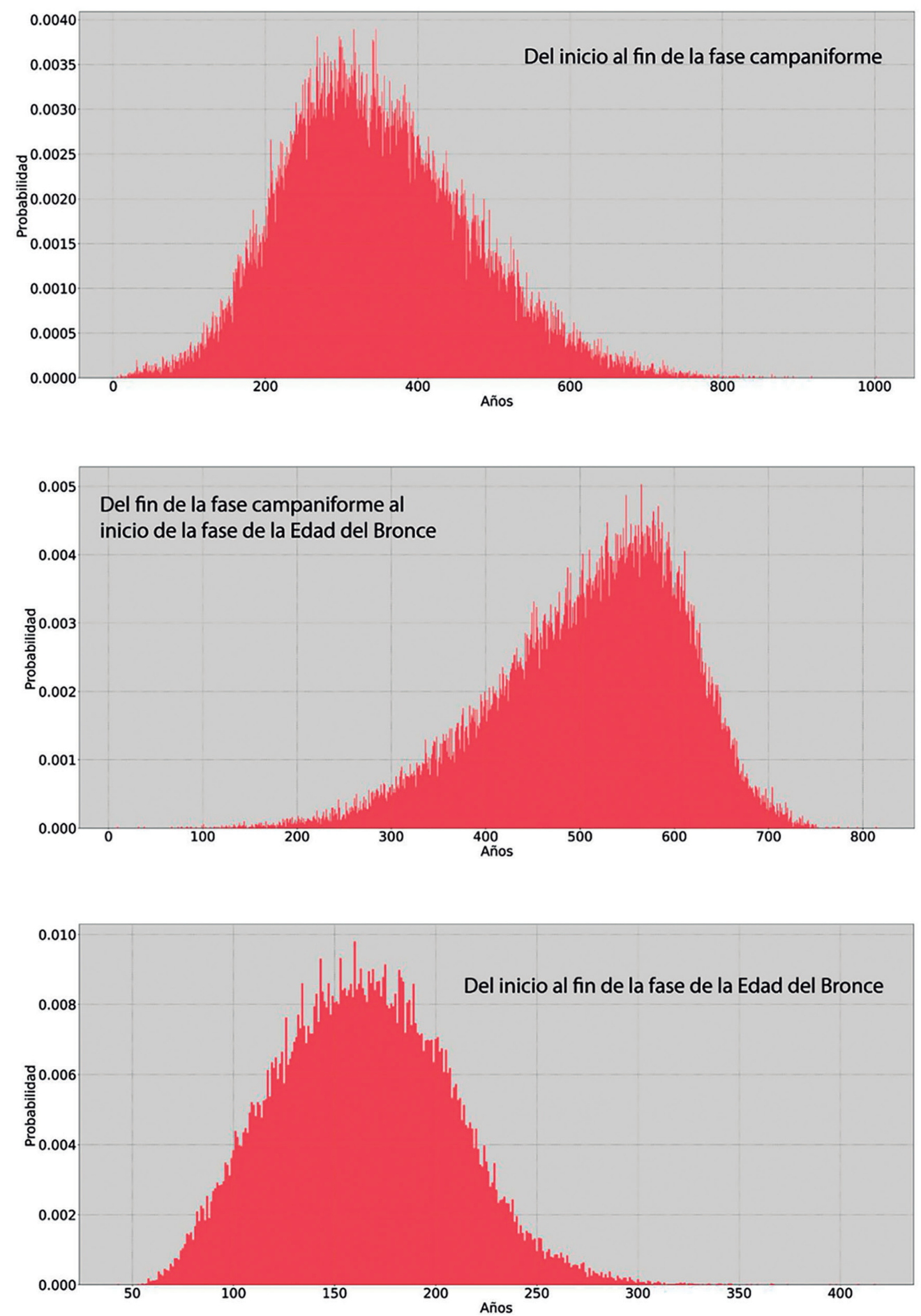

Fig.5. Gráficos de densidad de la probabilidad a posteriori para intervalos de tiempo entre límites. / Posterior density plots for the time elapsed between parameters. 
vada $(0,65)$ de que el individuo 1 fuera depositado más tarde que el 2 (arqueológicamente considerado una reducción), lo que sí podría indicar cierta diacronía en la deposición de los cuerpos. (Tabla 5)

Para la fase de la Edad del Bronce, los resultados son algo más precisos. Significativo es sin duda el periodo de unos 400 a 600 años, (410-640 tomando los límites del $80 \%$ de probabilidad) entre la fase campaniforme y la Edad Bronce, en el que no parece registrarse ninguna actividad funeraria, lo que supone al menos 16 generaciones sin enterramientos constatados.

Esta fase tiene un inicio en el intervalo 1630-1570 cal BC y un final, aún más ajustado en el intervalo 14701410 cal BC. La fase de la Edad del Bronce, durante 110-220 años (con un 80\% de probabilidad), reúne el mayor conjunto de enterramientos. Están distribuidos por distintos sectores del yacimiento y en cierta posición perimetral respecto a los previos sin observarse diferentes arcos temporales entre agrupaciones. Para las tumbas LM.459, con 2 de sus 5 individuos datados, y LM.283, con 3, se poseen indicadores de diacronía caracterizados por valores de probabilidad algo moderados. Sólo en el caso de la relación entre. LM.283(4) y LM.283(1) tenemos valores de probabilidad superiores a 0,6 . Es relevante tratándose de fosas simples, pues pudieron tener usos sucesivos más habituales de lo atribuido a este tipo de contenedores.

\begin{tabular}{|l|l|l|}
\hline Relación entre eventos & Probabilidad & Fase \\
\hline LM.211 (2) más reciente que LM.211 (1) & 0,48 & Campaniforme \\
\hline LM.461 (1) más reciente que LM.461 (2) & 0,65 & Campaniforme \\
\hline LM.459 (1) más reciente que LM.459 (4) & 0,56 & Edad del Bronce \\
\hline LM.283 (4) más reciente que LM.283 (2) & 0,54 & Edad del Bronce \\
\hline LM.283 (4) más reciente que LM.283 (1) & 0,63 & Edad del Bronce \\
\hline LM.283 (2) más reciente que LM.283 (1) & 0,59 & Edad del Bronce \\
\hline
\end{tabular}

Tabla 5: Estimación de la probabilidad para relaciones cronológicas entre distintos eventos (individuos) a partir del modelo bayesiano generado para Las Mayores. / Probability estimation for chronological relations between different events (individuals) from the Bayesian model of Las Mayores.

\subsection{Comparación entre las secuencias de los sitios}

El resultado de la función BchronDensity proporciona un gráfico (Fig. 6) del que ofrecemos un comentario general. Para Las Mayores los resultados son coherentes con las interpretaciones de las fases que ha ofrecido el modelo bayesiano. Frente a Las Mayores, las fechas de Camino de las Yeseras muestran la ocupación del enclave desde comienzos del III milenio cal $\mathrm{BC}$, próxima a Humanejos. Posiblemente en ambos, aunque no disponemos de fecha del foso Humanejos, una primera fase de actividad queda patente en sus recintos que, en el caso del primero de ellos, llegan hasta el 2500-2460 cal BC (Balsera et al., 2015a: 153).

Este momento coincide con el comienzo del registro funerario de Las Mayores y las tumbas asociadas a campaniforme en Humanejos y Camino de las Yeseras (Blasco et al., 2019; Garrido et al., 2019), que se acompañan de otras tantas sin la selecta cerámica.

El intervalo de duración del Campaniforme parece más largo en los yacimientos madrileños que en Las Mayores, a pesar de la imprecisión probabilística. En Camino de las Yeseras, la fecha Ua-35021: $3525 \pm 40$ BP (Gómez et al. 2011) (entre el 2006 -1707 cal BC) corresponde a uno de los pocos enterramientos primarios campaniformes del yacimiento.

Los modelos de ambos enclaves madrileños muestran su continuidad durante los comienzos de la Edad del Bronce, pero también una cierta inflexión en el cambio de milenio que es aún más evidente en Las Mayores, sin un solo evento funerario fechado en ese lapsus de tiempo. El modelo para el conjunto de la región, incluso evitando redundancia, resalta este mismo aspecto, sin duda, en relación con la transición Calcolítico-Edad del Bronce.

Por el contrario, en esta cronología comienza la ocupación de Los Berrocales que se extiende por toda la Edad del Bronce. Durante el Bronce medio, las fechas constatan una ocupación paralela de Las Mayores y Los Berrocales. Una única fecha procedente de un contexto doméstico (Aliaga y Megías, 2011: 170) (806-1004 cal BC) permite plantear en Los Berrocales una ocupación hasta el Bronce final.

\section{DISCUSIÓN}

La incompleta delimitación y excavación del yacimiento de Las Mayores es la primera cuestión a tener en cuenta en la evaluación de su episodio temporal sin enterramientos como un posible hiatus. Desconocemos la extensión total del yacimiento por lo que no es factible cuantificar el porcentaje de lo excavado frente al total, ni asegurar una completa documentación funeraria. En poblados calcolíticos peninsulares extensos y en los que se ha desarrollado una actividad arqueológica más intensa como Valencina de la Concepción, en Sevilla, se ha probado la inexistencia de delimitación entre los espacios domésticos y funerarios (Cintas et al., 2018). En el propio Humanejos las tumbas campaniformes se agrupan en dos sectores diferenciados (Garrido-Pena et al., 2019:19). Para la etapa del Bronce medio, hasta el momento, no conocemos una norma de situación de las tumbas, únicamente se observan agrupaciones (Barroso et al., 2014) cuyo carácter único o múltiple habrá que determinar.

Por otro lado, tanto Humanejos, como Camino de las Yeseras tienen un importante número de tumbas previas y contemporáneas al Campaniforme, aun sin los ajuares característicos, que no tiene parangón en Las Mayores. Coinciden los tres, sin embargo, en el número de tumbas asociadas a Campaniforme que, como en otros contextos, no es muy elevado (Barroso et al., 2015; Bueno et al., 2008). En ninguno de ellos superan la decena y en conjunto el número de individuos enterrados no sobrepasa 


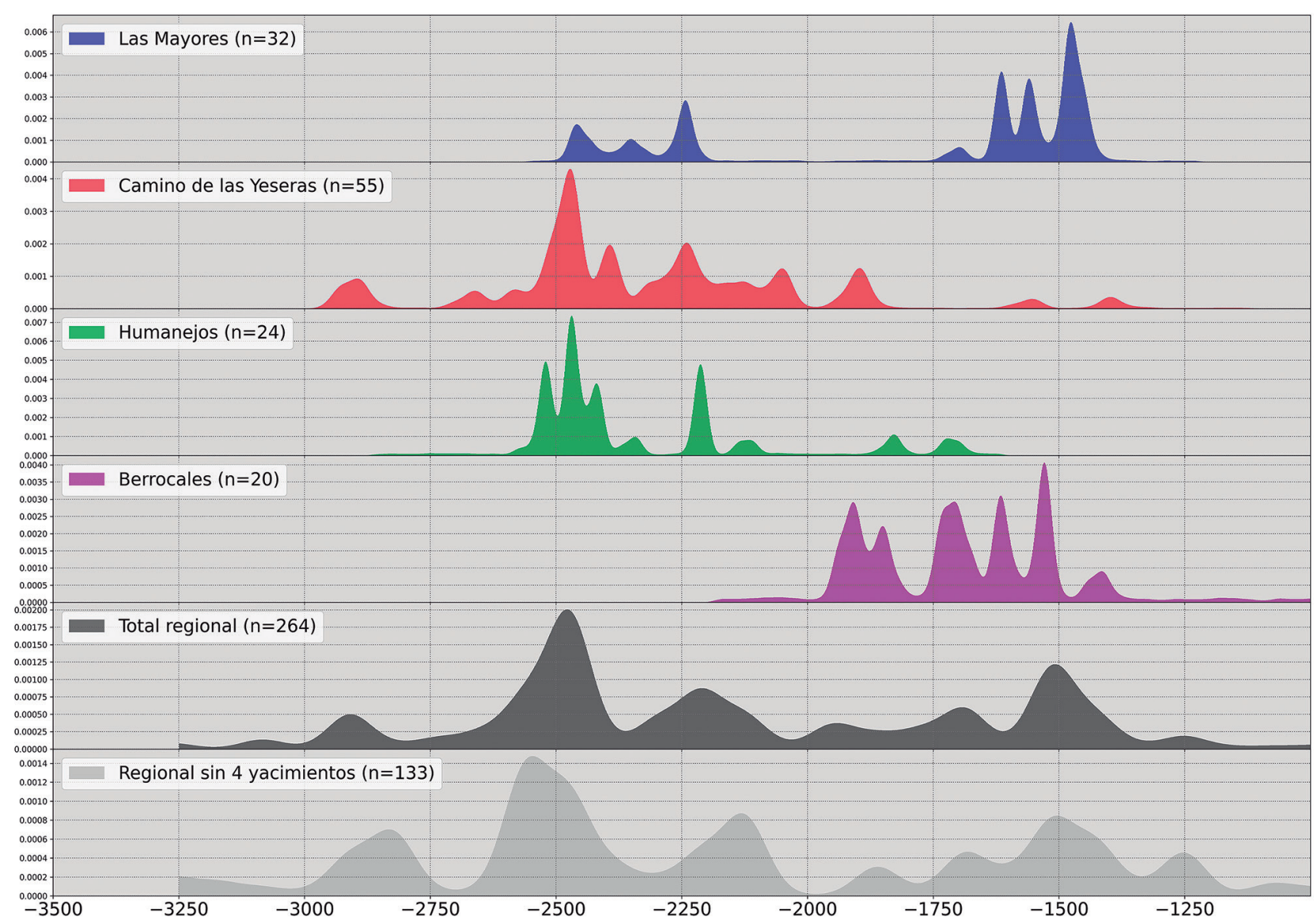

Fig.6. Gráficos de densidad de la probabilidad realizados con BchronDensity, de la biblioteca Bchron para los sitios de Las Mayores, Camino de las Yeseras, Humanejos, Berrocales y la región. / Density of probability plots rendered by BchronDensity, from Bchron library, for Las Mayores, Camino de las Yeseras, Humanejos, Berrocales sites and the region.

la veintena (Garrido-Pena et al., 2019 Liesau et al., 2018). En este punto, el registro de Las Mayores parece menos parcial. Por tanto, el lapso temporal del campaniforme en el yacimiento contribuye a determinar su auge en la zona, con independencia de fechas de cronología más reciente que prolongan su declive.

Es difícil precisar si para entonces se siguen fabricando cerámicas campaniformes o utilizando esos mismos espacios funerarios. Un buen ejemplo es la tumba 9 de Humanejos con al menos una fecha reciente (Ua41491: 3679 \pm 35 BP) (entre el 2176 y 1953 cal BC) y evidencias de su destrucción intencionada en época campaniforme (Garrido-Pena et al., 2019:23). En todo caso, la prolongación del Campaniforme en la Meseta ya ha sido destacada en varias ocasiones y cuenta con más contextos (Bueno et al., 2017). No hay que ignorar la capacidad de la población de Camino de las Yeseras o Humanejos de atraer riqueza, pues el oro o el marfil están presentes en muchas tumbas campaniformes (Garrido-Pena et al., 2019; Liesau, 2016;) en comparación con Las Mayores. Incluso, el mayor tamaño de ambos yacimientos (no necesariamente ocupados siempre con la máxima extensión) justificaría su prolongada filiación calcolítica.
Al margen del campaniforme, la discontinuidad en las dataciones percibida en Las Mayores es menor en Camino de las Yeseras y Humanejos. De poderse equiparar esa discontinuidad en pérdida de población, lo hacen progresivamente y aún tienen ocupación, o cronologías que la sostienen, en el momento en que nuevos poblados como Los Berrocales emergen en la región. En los enterramientos de Los Berrocales no hay registro alguno de Campaniforme (Aliaga y Megías, 2011: 175) y en todos ellos están ausentes los ítems de prestigio, siendo sólo la cerámica o adornos en conchas locales los únicos elementos asociados, raramente, a los difuntos. Que los enterramientos de Los Berrocales fechados hasta ahora se sitúen en el Bronce antiguo y medio, coincidiendo estos últimos con los de Las Mayores, sin prolongarse más, tampoco parece casual. Los enterramientos Cogotas del valle del Tajo (Barroso et al., 2014), igual que en la Meseta Norte (Esparza et al., 2012), son menos numerosos frente a los de la etapa previa Protocogotas.

Ciertamente, una interpretación razonable a la coincidencia de falta de registro de actividad de Las Mayores y el inicio de ocupación de Los Berrocales es la existencia de una contracción de su población en pa- 
ralelo a una reorganización de las comunidades regionales, con traslados graduales. La atracción de parte de la población calcolítica hacia enclaves que buscan una cierta altura es algo que ya se ha planteado (Ríos et al., 2012; Blasco et al., 2019), aun sin abandonarse los medios fluviales, como prueba Humanejos.

Los resultados de los estudios de isótopos realizados en la Comunidad de Madrid, incluyendo a 35 individuos de Los Berrocales, sugieren una movilidad regional, entre la sierra y las zonas bajas, e interregional reducida. Solo cuatro individuos eran inmigrantes, tres de ellos mujeres quizás relacionadas con patrones de exogamia (Díaz del Rio et al., 2017b). Tres de ellas, dos de Los Berrocales y una más del yacimiento de Pista de Motos se sitúan en el Bronce medio a partir de fechas o su asociación a materiales Cogotas I.

Por otro lado, el estudio genómico de Camino de las Yeseras y Humanejos ha revelado la presencia de población de origen local y foráneo (Olalde et al., 2018). De los 12 individuos muestreados en Humanejos, tres asociados a campaniforme y uno posterior de la Edad del Bronce presentan ancestría centroeuropea. Su patrón genético, similar a otros individuos peninsulares analizados, apunta la llegada episódica de individuos procedentes de Centroeuropa, posiblemente desde la $2^{\circ}$ mitad del III milenio cal BC., que conviven con las poblaciones locales (Olalde et al., 2019; Garrido-Pena et al., 2019: 231).

\section{CONCLUSIONES}

Los yacimientos característicos del interior peninsular son poblados permanentes. Sin embargo, forman complejos palimpsestos que superponen en el mismo espacio estructuras de ocupaciones diacrónicas cuya caracterización final equipara continuidad y recurrencia. Afinar su caracterización, mostrando interrupciones o los máximos y mínimos de su ocupación, conlleva dificultades. El análisis cronológico de Las Mayores lo pone de manifiesto. La secuencia cronológica de la que disponemos hasta el momento se interrumpe a diferencia de otras ocupaciones del entorno. El modelo bayesiano nos permite precisar la importancia temporal de la interrupción. Sin embargo, la cuestión cambia cuando analizamos los resultados de Las Mayores en su entorno inmediato y con yacimientos contrastables.

En un área relativamente reducida las cronologías plantean dos modelos para el final de los poblados calcolíticos, el brusco abandono (aunque no permanente en Las Mayores) o una paulatina desarticulación y transformación de sus comunidades. Nuestro análisis se ha limitado a las fechas, sin indagar en la problemática cultural ya tratada en distintos ámbitos (por ejemplo, Valera, 2003 y 2015). Sin duda el área analizada exige de más fechas y excavaciones en extensión antes de concluir sobre una única o múltiple respuesta de movilidad y las causas que hay detrás.
Las fechas obtenidas en Las Mayores muestran una discontinuidad, pero su excavación parcial no permite asegurar que el poblado sea abandonado y reocupado, casi medio siglo después, por otra población. En paralelo, los yacimientos de la región tienen mayor continuidad, si bien es cierto que el cambio de milenio implica una contracción de su ocupación que podría ser más difícil de detectar en Las Mayores. Naturalmente el tamaño de los asentamientos, el medio que los sostiene, la población agrupada en ellos son factores a tener en cuenta en la dinámica interna de cada yacimiento. La simultánea emergencia de nuevos poblados sugiere una reestructuración poblacional que, por los datos genéticos y de movilidad, contó solo con ocasional aporte externo. La fisionomía de poblados como Los Berrocales, a modo de extensión fraccionada con diferentes agrupaciones de estructuras, se presta a esa idea de anexos.

Las características de los poblados de hoyos dificultan la obtención de datos paleoeconómicos que son importantes y una tarea aún pendiente en Las Mayores. No debe dejarse de lado que la discontinuidad de su registro coincide con la transición del Calcolítico a la Edad del Bronce caracterizada, en ocasiones, como abrupta (Blanco et al., 2018), y relacionada con el potencial impacto del evento climático 4.2 ka cal BP (ca. 2350-1850 cal BC) (Magny et al., 2009). Dicho episodio es considerado relevante en la Meseta Sur (López et al., 2017) aunque es difícil determinar una coincidencia temporal clara y la severidad del impacto, que no solo afectaría a Las Mayores.

En todo caso, cualquier repercusión medioambiental habría quedado reparada en el Bronce medio en el que el yacimiento toledano constata una importante ocupación. Conviene destacar la treintena de individuos documentada cuando los enterramientos escasean en el conjunto de la Meseta (Esparza et al., 2012; Barroso et al., 2014). Habrá que determinar si este importante registro de Las Mayores, Humanejos, Los Berrocales e incluso los visos de movilidad que apuntan a prácticas exogámicas coinciden, en este Bronce medio, con un periodo demográfico álgido en la región.

\section{AGRADECIMIENTOS}

El estudio de Las Mayores se ha realizado con sucesivos proyectos financiados por la JCCM y la UAH (SBPLY/17/180801/000068; SBPLY/18/180801/000083) y gracias a la disposición de D. Juan Manuel Rojas que excavó el yacimiento. Para la obtención de muestras obtuvimos permiso del Servicio de Patrimonio y Arqueología de Castilla - La Mancha y el Museo de Santa Cruz, Toledo. Agradecemos a su director y conservadores toda la ayuda prestada. A Marcos Sáez y el profesor Dr. Oscar Cambra (UAM) les agradecemos sus aportaciones en el estudio de los restos antropológicos. 


\section{BIBLIOGRAFÍA}

Aliaga, R., Megías, M., 2011. Los Berrocales (Madrid): Un yacimiento de la Edad del Bronce en la confluencia Manzanares-Jarama. Universidad Autónoma de Madrid, Madrid.

Alpoim Guedes, J. de, Manning, S. W., Bocinsky, R. K., 2016. A 5,500-year model of changing crop niches on the Tibetan Plateau. Current Anthropology 57(4), 517-522.

Aranda, G., Díaz-Zorita, M., Hamilton, D., Milesi, L., Sánchez Romero, M., 2020. A radiocarbon dating approach to the deposition and removal of human bone remains in megalithic monuments. Radiocarbon 62(5),1147 - 1162.

Arteaga, C., Liesau, C. García, R., Pérez, E., Menduiña, R., Vega, J., Blasco, C., 2017. The Ditched Enclosure of Camino de las Yeseras (Madrid): A Sedimentological Approach to the Study of some Singular Structures. Espacio, Tiempo y Forma Series I-VII 10, 77-94.

Bailey, G., 2007. Time perspectives, palimpsests and the archaeology of time. Journal of Anthropological Archaeology 26, 198-223.

Balsera, V., Díaz del Rio, P., 2014. Haciendo tiempo. La cronología absoluta de la Prehistoria reciente madrileña. En: Actas de las novenas Jornadas de Patrimonio Arqueológico en la Comunidad de Madrid, 35-50. Comunidad de Madrid.

Balsera, V., Bernabeu-Aubán, J., Costa-Caramé, M., Diáz-delRío, P., García-Sanjuán, L., Pardo, S., 2015a. The radiocarbon chronology of southern Spain's late prehistory (5600-1000 cal BC): A comparative review. Oxford Journal of Archaeology 34(2), 139-156.

Balsera, V., Díaz-del-Río, P., Gilman, A., Uriarte, A., Vicent, J. M., 2015b. Approaching the demography of late prehistoric Iberia through summed calibrated date probability distributions (7000-2000 cal BC). Quaternary International 386, 208211.

Bernabeu Aubán, J., García Puchol, O., Barton, M., McClure, S., Pardo, S., 2016. Radiocarbon dates, climatic events, and social dynamics during the Early Neolithic in Mediterranean Iberia. Quaternary International 403, 201-210.

Barroso, R., Bueno, P., Balbín, R., González, A., Rojas, J.M., 2018b. Inhumaciones en urna cerámica de la Edad del Bronce en la cuenca media y alta del Tajo. Una perspectiva comparativa. Spal 27,13- 30.

Barroso, R., Bueno, P., González, A., Balbín, R. de; Rojas, J.M., 2018a. Tumbas, materialidad y maternidad en los enterramientos de mujeres con Campaniforme: Dos casos de estudio del valle medio del Tajo. Complutum 29(2), 319-337.

Barroso, R., Bueno, P., González, A., Candelas, N., Rojas, J.M., López, J.A., 2014. Enterramientos de la Edad del Bronce en la Meseta Sur peninsular a partir del Sector 22, Yuncos (Toledo). Munibe Antropologia-Arkeologia 65, 117-136.

Barroso, R., Bueno, P., Vázquez A., González, A., Peña, L., 2015. Enterramientos individuales y enterramientos colectivos en necrópolis del megalitismo avanzado del interior: la cueva 9 de Valle de las Higueras. Toledo. In: Death as Archeology of transition: thoughts and materials, 165-176. Bar International series 2078, Oxford.

Blanco-González, A., Lillios, K.T. López-Sáez, J.A., Drake, B.L., 2018. Cultural, Demographic and Environmental Dynamics of the Copper and Early Bronze Age in Iberia (3300-1500 BC): Towards an Interregional Multiproxy Comparison at the Time of the 4.2 ky BP Event. Journal of World Prehistory 31(1), 1-79.
Blasco, C., Delibes, G., Baena, J., Liesau, C., Rios, P., 2007. El poblado calcolítico de Camino de las Yeseras (San Fernando de Henares, Madrid): un escenario favorable para el estudio de la incidencia campaniforme en el interior peninsular. Trabajos de Prehistoria 64(1), 151-163.

Blasco, C., Liesau, C., 2019. Mundos paralelos: la convivencia de otras prácticas funerarias con los rituales campaniformes. In Delibes, G., Guerra, E., (Eds.), ¡Un brindis por el príncipe! El vaso campaniforme en el interior de la Península Ibérica (2500-2000 a.C.), vol. I, 340-363. Museo Arqueológico Regional, Madrid

Blasco, C., Liesau, C. Rios, P., 2019. El registro funerario campaniforme de la Región de Madrid reflejo de una sociedad plural y compleja. In Delibes, G., Guerra, E., (Eds.), ¡Un brindis por el príncipe! El vaso campaniforme en el interior de la Península Ibérica (2500-2000 a.C.), vol. I, 247-277. Museo Arqueológico Regional, Madrid.

Blasco, C., Montero, I., Flores, R., 2016. Bell Beaker funerary copper objects from the center of the Iberian Peninsula in the context of the Atlantic connections. In: Guerra, E., Liesau, C., (eds.), Analysis of the Economic Supporting the Social Supremacy the Beaker Groups Proceedings of the XVII UISPP World Congress, 19-35. Archaeopress, Oxford.

Buck, C.E., Christen J.A., James G.N., 1999. BCal: an on- line Bayesian radiocarbon calibration tool. Internet Archaeology, 7.

Buck, C.E., Meson, B., 2015: On being a good Bayesian. World Archaeology 47(4), 1-18.

Bueno-Ramírez, P., Barroso, R., Balbín, R., 2008. Campaniforme en las construcciones hipogeas del megalitismo reciente al interior de la Península Ibérica. Veleia 24-25, 771-790.

Bueno-Ramírez, P., Barroso, R., Balbín, R., 2017. Redefining Ciempozuelos Bell-Beaker culture in Toledo? In: Gonçalves, V., (ed.), Sinos e taças. Junto ao océano e mais longe. Aspectos da presença campaniforme na península ibérica, 324-341. Uniarq, Lisboa.

Cintas-Peña, M., García Sanjuán, L., Díaz-Zorita, M., Herrero, A.M., Robles, S., 2018. The non-adult population at the Copper Age settlement of Valencina de la Concepción (Seville, Spain): a demographic, contextual and sociological approach. Trabajos de Prehistoria 75(1), 85-108.

Díaz-del-Río, P., 2001. La Formación del Paisaje Agrario: Madrid en el III y II milenios AC. Comunidad de Madrid, Madrid.

Díaz-del-Río, P., 2003. Recintos de fosos del III milenio AC en la Meseta peninsular. Trabajos de Prehistoria 60(2), 61-78.

Díaz-del-Río, P., Consuegra, S., Audije, J., Zapata, S., Cambra, O., González, A., Waterman, A., Thomas, J., Peate, D., Odriozola, C., Villalobos, R., Bueno, P., Tykot, RH., 2017a. Un enterramiento colectivo de mediados del III milenio AC en el centro de la Península Ibérica: El Rebollosillo (Torrelaguna, Madrid). Trabajos de Prehistoria 74(1), 68-85.

Díaz-del-Río, P., Vicent, J., 2006. Movilidad, funcionalidad y usos del suelo en la Prehistoria Reciente. Arqueología Espacial 26: $21-36$

Díaz-del-Río, P., Waterman, A. J., Thomas, J. T., Peate, D. W., Tykot, R. H., Martínez-Navarrete, M. I., Vicent, J. M., 2017b. Diet and mobility patterns in the Late Prehistory of central Iberia (4000-1400 cal BC): the evidence of radiogenic (87Sr/86Sr) and stable $(\delta 180, \delta 13 \mathrm{C})$ isotope ratios. Archaeological and Anthropological Sciences 9, 1439-1452. 
Esparza, A., Velasco, J., Delibes, G., 2012. Hum 2005-00139: Planteamiento y primeros resultados de un proyecto de investigación sobre la muerte en Cogotas I. In: Rodríguez Marcos, J.A., Fernández Manzano, J., (eds.), Cogotas I. Una cultura de la Edad del Bronce en la Península Ibérica, 259-320. Universidad de Valladolid, Valladolid.

Galindo, I., Marcos, V., Montero, I., 2018. Metalurgia en el yacimiento de "La Serna", en Arganda del Rey. In Actas Reunión de Arqueología madrileña 2016, 149-159. Colegio oficial de Doctores y licenciados en Filosofía y Letras, Madrid.

García-Martínez de Lagrán, I., Tejedor, C., 2019. "Tiempos" de Campaniforme: análisis cronométrico de las dataciones radiocarbónicas procedentes de contextos con cerámica campaniforme en el yacimiento de Humanejos (Parla, Madrid). In: Garrido, R., Flores, R., Herrero, A.M., (Coors.), Las sepulturas campaniformes de Humanejos (Parla, Madrid), 322-333. Comunidad de Madrid, Madrid.

Garrido-Pena, R., Flores, R., Herrero, A.M., (Coords.), 2019. Las sepulturas campaniformes de Humanejos (Parla, Madrid). Comunidad de Madrid, Dirección General de Patrimonio Cultural, Madrid.

Gómez, J.L., Blasco, C., Trancho, G., Ríos, P., Grueso, I., Martínez, M.S., 2011. Los protagonistas. In: Blasco, C., Liesau, C., Ríos, P., (Eds.), Yacimientos calcolíticos con campaniforme de la región de Madrid: nuevos estudios, 101-132. Patrimonio arqueológico de Madrid 6 Universidad Autónoma de Madrid, Madrid.

Hamilton, W. D., Krus, A. M., 2017. The myths and realities of Bayesian chronological modelling revealed. American Antiquity 83(2), 187-203.

Haslett, J., Parnell, A., 2008. A simple monotone process with application to radiocarbon-dated depth chronologies. Journal of the Royal Statistical Society: Series C (Applied Statistics) 57(4), 399-418.

Jover Mestre, F.J., López Padilla, J.A., García-Donato Layrón, G., 2014. Radiocarbono y estadística bayesiana: aportaciones a la cronología de la Edad del Bronce en el extremo oriental del sudeste de la Península Ibérica. Sagvntvm (PLAV) 46, 41-69.

Liesau, C., 2016. Some prestige goods as evidence of interregional interactions in the funerary practices of the Bell Beaker groups of Central Iberia. In: Guerra, E., Liesau, C. (eds.), Analysis of the Economic Supporting the Social Supremacy the Beaker Groups Proceedings of the XVII UISPP World Congress, 69-94. Archaeopress, Oxford.

Liesau, C., Ríos, P., Blasco, C., Ortíz, I., 2018. Dentro y fuera de las tumbas campaniformes en Camino de las Yeseras: ¿una segunda vida para los muertos? Anejos a Cuadernos de la Universidad Autónoma de Madrid 3, 141-152.

López- Sáez, J. A., Blanco, A., Pérez-Díaz, S., Albaz, F., Luelmo, R., Glais, A. y Nuñez, S., 2017. Landscapes, human activities and climate dynamics in the South Meseta of the Iberian Peninsula during the 3rd and 2nd millennia calBC. In: Bartelheim, M., Bueno, P., Kunst, M. (Eds.), Key resources and socio-cultural developments in the Iberian Chalcolithic, 129-142. Eberhard Karls Universität Tübingen, Tübingen.

Magny, M., Vannière, B., Zanchetta, G., Fouache, E., Touchais, G., Petrika, L., Coussot, C., Walter-Simonnet, A-V., Arnaud, F., 2009. Possible complexity of the climatic event around 4300$3800 \mathrm{cal}$. BP in The central and western Mediterranean. The Holocene 19, 823-833.

McLachlan, G., Peel, D., 2000. Finite Mixture Models. Nueva York, John Wiley \& Sons.
Olalde, I., Brace, S., Allentoft, M. y más, 2018. The Beaker phenomenon and the genomic transformation of northwest Europe. Nature 555, 190-196.

Olalde, I., Mallick, S.,Patterson, N., y más, 2019. The genomic history of the Iberian Peninsula over the past 8000 years. Science 15 Vol. 363, Issue 6432, 1230-1234.

Orton, D., Gaastra, J., Vander Linden, M., 2016. Between the Danube and the Deep Blue Sea: Zooarchaeological Meta-Analysis Reveals Variability in the Spread and Development of Neolithic Farming across the Western Balkans. Open Quaternary 2, 6. DOI: http://doi.org/10.5334/oq.28

Perera, J., Garrido, G., Pérez, J., Rojas, J.M., 2010. La excavación arqueológica en el yacimiento las Mayores en Numancia de la Sagra (Toledo) Campaniforme y Bronce en la Comarca de la Sagra. In: Madrigal, A., Perlines, M., (Coords.), Actas de las II Jornadas de arqueología de Castilla - La Mancha (Toledo 2007). CD. Diputación de Toledo - Castilla La Mancha, Toledo.

Pérez Villa, A., 2015. Pautas funerarias y demográfica de la Edad del bronce en la cuenca media del Tajo. Bibliotheca praehistorica hispana 31, Madrid.

Philippe, A. Vibet, M.A., 2020. Analysis of Archaeological Phases Using the R Package ArchaeoPhases. Journal of Statistical Software, 93.

Ramsey, C.B., 2009. Bayesian analysis of Radiocarbon dates. Radiocarbon 51(1), 337-360.

Reimer, P., Austin, W., Bard, E., Bayliss, A., Blackwell, P., Bronk, C. y otros, 2020. The IntCal20 Northern Hemisphere Radiocarbon Age Calibration Curve (0-55 cal kBP). Radiocarbon 62(4), 725-757.

Ríos, P., 2011. Territorio y sociedad en la región de Madrid durante el III milenio AC. El referente del yacimiento de Camino de las Yeseras, Patrimonio Arqueológico de Madrid 7. Universidad Autónoma de Madrid, Madrid.

Ríos, P., Blasco, C., Aliaga, R., 2012. Entre el Calcolítico y la Edad del Bronce. Algunas consideraciones sobre la cronología campaniforme. Cuadernos de Prehistoria y Arqueología de la UAM 37-38, 195-208.

Shennan, S., 1988. Quantifying archaeology, University press, Edinburgh.

Valera, C., 2003. Problemáticas do abandono no povoamento Calcolitico do ocidente peninsular. ERA Arqueologia 5, 126-148.

Valera, C., 2015. Social change in the late 3rd millennium BC in Portugal: the twilight of enclosures. In: Meller, H., Arz, H.W., Jung, R., Risch, R. (Eds.), 2200 BC - A climatic breakdown as a cause for the collapse of the Old World?, 409-428. Landesmuseum fu"r Vorgeschichte, Halle. 